\title{
Background Rejection in the ARA Experiment
}

\author{
Carl Pfendner ${ }^{1, \star}$ for the ARA collaboration \\ ${ }^{1}$ Dept. of Physics and CCAPP, The Ohio State University, 191 W. Woodruff Ave., Columbus, OH 43210, \\ USA
}

\begin{abstract}
The Askaryan Radio Array (ARA) is a radio frequency observatory under construction at the South Pole that is searching for ultrahigh energy neutrinos via the Askaryan effect. Thermal fluctuations currently dominate the trigger-level background for the observatory and anthropogenic sources also introduce a significant source of noise. By taking advantage of the observatory's regular geometry and the expected coincident nature of the RF signals arriving from neutrino-induced events, this background can be filtered efficiently. This contribution will discuss techniques developed for the ARA analyses to reject these thermal signals, to reject anthropogenic backgrounds, and to search for neutrino-induced particle showers in the Antarctic ice. The results of a search for neutrinos from GRBs using the prototype station using some of these techniques will be presented.
\end{abstract}

\section{Introduction}

The Askaryan Radio Array (ARA) aims to measure the flux of ultra-high energy (UHE) neutrinos above $10^{17} \mathrm{eV}$. While UHE neutrinos are so far undetected, they are expected both as decay products from the GZK process [1], as first pointed out by Berezinsky and Zatsepin [2], and directly from astrophysical sources, such as Gamma Ray Bursts (GRBs).

The interaction of a UHE neutrino in dense media induces an electromagnetic shower which in turn creates impulsive radiofrequency (RF) Cherenkov emission via the Askaryan effect [3] In radio transparent media, these RF signals can then be observed by antenna arrays read out with $\sim \mathrm{GHz}$ sampling rates.

Due to the $\sim 1 \mathrm{~km}$ radio attenuation lengths in ice [4, 5], radio arrays have the potential to view the $100 \mathrm{~s}$ of $\mathrm{km}^{3}$ of ice necessary to reach the sensitivity to detect $\sim 10$ events per year from expected UHE neutrino fluxes. Next-generation detectors are under construction aiming to reach the $100 \mathrm{~s}$ of $\mathrm{km}^{3}$ target volume of ice. The Askaryan Radio Array (ARA) [4] is one such detector being deployed in the ice at the South Pole and the results for a search from neutrinos from Gamma Ray Bursts using data from a prototype station of this detector are presented in these proceedings. However, the radio background is considerable and, in order to perform such a search, methods to reject thermal and anthropogenic sources of noise must be developed.

\footnotetext{
^e-mail: pfendner.1@osu.edu
} 


\section{ARA Testbed Signal Algorithms}

A number of background rejection methods have been previously developed for the ARA Testbed station [6]. Among these methods, two primary techniques were used to search for a diffuse flux of neutrinos: (1) requirements placed on the contents of an interferometric map (see Fig. (1)) and (2) a cut on the correlation between the peak signal strength and the cross-correlation of the aforementioned map (see Fig. (2)).
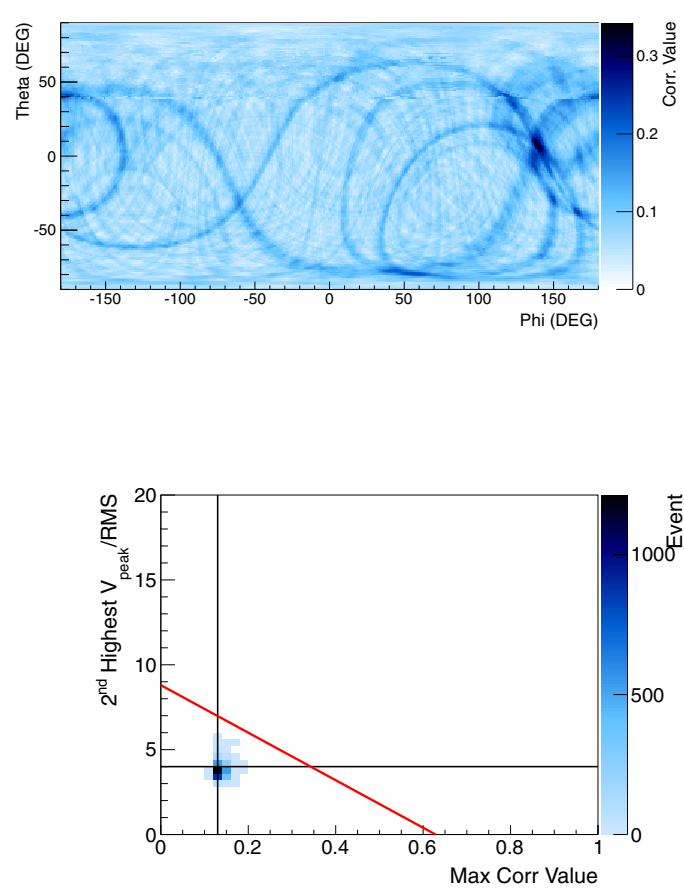

Figure 1. An interferometric map produced for a calibration pulser event. The peak of the map corresponds to the correct location of the calibration pulser in this 30-m radius map. The two criterion for the cuts based on this map are (1) that the peak is well-defined and (2) no other part of the map shows a comparable correlation strength. These are found by calculating the area of the $85 \%$ contour around the peak and the total area of comparable $85 \%$ strength on the rest of the map. The area around the peak $A_{\text {peak }}$ must be less than a given cut value and the ratio of $A_{\text {total }} A_{\text {peak }}$ must be less than a different given value.

Figure 2. This pair of plots shows the application of the Peak/Correlation cut. On the left is the set of events from the $10 \%$ burn sample of the full Testbed data set remaining after all other cuts have been applied. On the right is a set of $10^{18} \mathrm{eV}$ simulated events under the same conditions. The second highest V/RMS value and the peak correlation value on the interferometric map are found for each event. The cut is performed by accepting only those events above the diagonal line on the plot. The position of this line is found by optimizing for the result that gives the best expected limit.

\section{ARA Testbed GRB Search}

As GRBs are a potential source of UHE neutrinos, we performed a search for neutrino events correlated with the Gamma-ray Coordinates Network (GCN)[7] catalog of GRBs, as found in the GRB-web database [8], occurring during the operational period of the ARA Testbed. Of the $\sim 500$ GRBs in the catalog, we limited our search to the 57 GRBs that coincide with known active high-quality datataking periods and were also found within the ARA Testbed's field of view (see Fig. (3)). Since GRBs 
are localized in time, we limited our signal search windows to the 10 minutes centered on each GRB event, using the hour before and the hour after the GRB as a background analysis period. We used a two-stage unblinding procedure. First $10 \%$ of the data in the background period was analyzed and the cuts optimized against these events. In the first unblinding, we apply the optimized cuts to the remaining $90 \%$ of the background analysis period and compare these results to the expectation from the $10 \%$ set. After confirmation of the expected results from the background period search, we then perform the second unblinding and apply the cuts to $100 \%$ of the events in the signal search window.
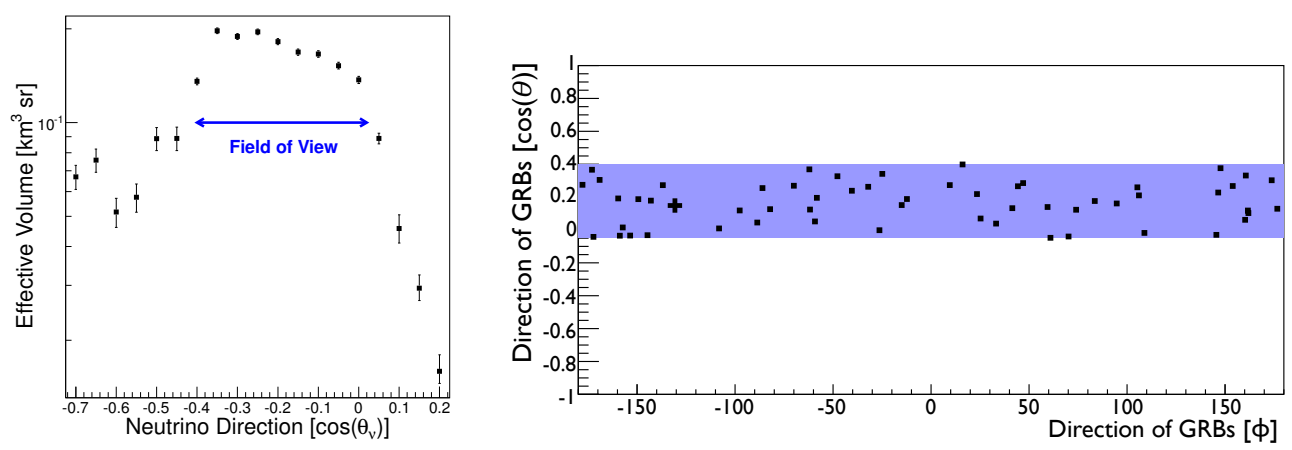

Figure 3. Left: Effective volume of the ARA Testbed as a function of the zenith angle $\left(\theta_{v}\right)$ of the neutrino travel direction with a neutrino energy of $10^{7.5} \mathrm{GeV}$. The field of view is defined as the Full Width Half Maximum (FWHM) of the effective volume, which is $-0.4<\cos \theta_{v}<0.05$. This field of view covers $\sim 20 \%$ of the sky. A vertically up-going neutrino has $\cos \theta_{v}=1$. Right: The distribution map of 57 selected GRBs in Testbed local coordinates. The blue band in the map is the field-of-view cut range defined in the left figure.

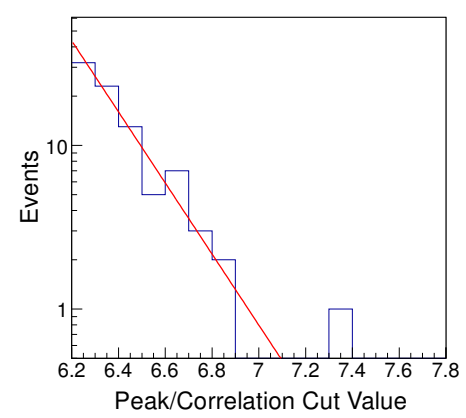

Figure 4. The differential distribution of events found in the background analysis windows of the $10 \%$ data set as a function of Peak/Correlation Cut Value after all other cuts have been applied. This distribution is fitted with an exponential function (diagonal red line) which is used to extrapolate the number of expected background events for a higher Peak/Correlation Cut Value. The optimized value is 7.6 .

For each GRB, we use the observed values found in the catalog to model the expected neutrino fluence using the NeuCosmA simulation package [9]. We optimize our cuts for the best expected limit based on a simulated neutrino sample generated from the average flavor-weighted flux of all 57 GRBs examined and the data collected during $10 \%$ of the background window. Using a fit to the differential distribution of events in the background set, we find the expected number of background events as a function of cut value (see Fig. (4p). After optimization we found that all three cuts that were adjusted were loosened (see Table 1) when compared with the diffuse search performed previously. The Delay 
Table 1. Comparison of cut parameter values of the analysis. See text for details.

\begin{tabular}{lcccc}
\hline Cut & Delay Difference Cut & \multicolumn{2}{c}{ Reconstruction Quality Cut } & Peak/Correlation Cut \\
\hline Parameter & On/Off & $A_{\text {peak }}$ & $A_{\text {total }} / A_{\text {peak }}$ & Peak/Corr. Cut Value \\
\hline Diffuse Neutrino Search & On & $<50 \mathrm{deg}^{2}$ & $<1.5$ & $>8.8$ \\
GRB Neutrino Search & Off & $<140 \mathrm{deg}^{2}$ & $<16.4$ & $>7.6$ \\
\hline
\end{tabular}

Difference cut was only optimized in whether the cut was used or not; this was due to the expectation that this cut was largely redundant based on the results of the diffuse search.

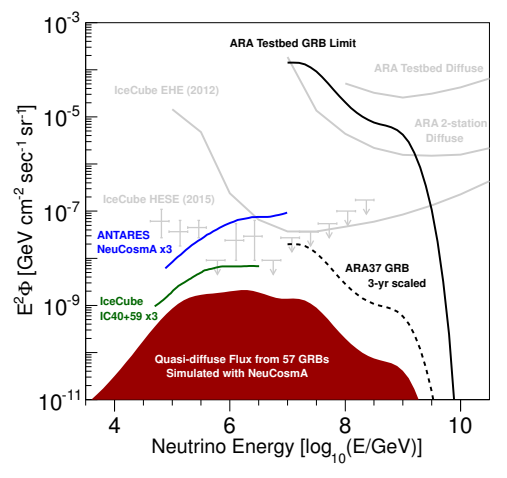

Figure 5. The ARA-Testbed quasi-diffuse all-flavor flux limit. This is the first quasi-diffuse flux limit above $10^{17} \mathrm{eV}$. Although the limit is not yet comparable to the limits placed at lower energies, applying scaling factors to the result predicts that a GRB quasi-diffuse limit from the full 37-station ARA will be competitive. Additional improvements at the trigger and analysis levels may improve these results even further.

\section{Conclusions}

Using interferometric cross-correlation maps and signal strength parameters, we have developed a number of effective background rejection techniques for the ARA detector. These methods are flexible enough to be adapted to perform multiple neutrino searches including a diffuse neutrino search and a search for neutrinos correlated with GRBs. Further development of these methods and the more regular geometry of deep stations will provide even greater benefits to further analyses.

\section{References}

[1] K. Greisen, Phys. Rev. Lett., 16, 748 (1966); G. T. Zatsepin and V. A. Kuzmin, JETP Lett., 4, 78 (1966)

[2] V. S. Berezinsky and G. T. Zatsepin, Phys. Lett., B28, 423 (1969); V. S. Berezinsky and G. T. Zatsepin, Sov. J. Nucl. Phys., 11, 111 (1970)

[3] G. A. Askaryan, JETP, 14, 441, (1962); G. A. Askaryan, JETP, 21, 658 (1965); P. Gorham et al., Phys. Rev., 62, 8590 (2000); D. Saltzberg et al., AIP Conf. Proc., 579, 225 (2001); P. W. Gorham et al., Phys. Rev., D72, 023002 (2005); P. W. Gorham et al., Phys. Rev. Lett., 99, 171101 (2007)

[4] Allison, P. et al., Astropart.Phys., 35, 457 (2012)

[5] Barwick, S. and Besson, D. and Gorham, P. and Saltzberg, D., J. Glaciol., 51, 231 (2005)

[6] P. Allison et al., Astroparticle Physics, 70, 0, 62 (2015)

[7] http://gcn.gsfc.nasa.gov

[8] http://icecube.wisc.edu/science/tools

[9] P. Baerwald, S. Hummer, and W. Winter, Phys. Rev, D83, 067303 (2011). 